\title{
Existentielle Erfahrungen am Berg
}

\section{Beatrice Sandberg \\ Universität Bergen/Norwegen}

Zusammenfassung: Unter Verwendung von intertextuellen Verweisen auf Ludwig Hohls "Bergfahrt" von 1975 greift Roman Graf das Motiv des zielstrebigen oder sturen Bergsteigers auf in seinem Roman "Niedergang", der mit seiner Freundin eine Gipfelbesteigung in Angriff nimmt, die während der Wanderung zunehmend katalysatorische Funktionen annimmt. Graf ist nicht der einzige der kritischen Autoren, die eine Tradition aufgreifen und umfunktionieren. Ob historisch verankert wie in Silvio Huonders "Die Dunkelheit in den Bergen" oder zivilisationskritisch motiviert wie Urs Augstburgers Bergtrilogie, die u.a. ein Zukunftsszenario über die Schrecken des Klimawandels enthält, oder aber ein Erstlingswurf wie Urs Mannharts "Luchs": diese Romane (und zahlreiche weitere, darunter auch Krimis) beleben eine Tradition, von der sie sich absetzen und die sie mit kritischen Elementen zugleich erneuern.

Schlüsselbegriffe: intertextuell, Verweise, Ludwig Hohl, Bergfahrt, Motiv, Bergsteiger, zielstrebig, stur, Gipfelbesteigung, Wanderung, katalysatorisch, Tradition, umfunktionieren, Roman, absetzen, kritisch

Resumo: Sob utilização de referências intertextuais da obra de Ludwig Hohl "Bergfahrt" de 1975, Roman Graf aborda no seu romance "Niedergang" o motivo do alpinista persistente ou teimoso, que enfrenta com a sua namorada uma ascensão ao cume que durante a caminhada assume progressivamente funções catalisadoras. Graf não é o único autor crítico que aborda uma tradição e a transforma. Tanto se trate de um romance historicamente ancorado como em Silvio Huonder "Die Dunkelheit in den Bergen" ou motivado por uma crítica de civilização como a triologia da montanha de Urs Augstburger, que, entre outros, contém um cenário futurista sobre o terror das alterações climáticas, ou então um primeiro esboço como o "Luchs" de Urs 
Mannhart: estes romances (e muitos outros, incluindo romances policiais) animam uma tradição de qual se destacam e a renovam ao mesmo tempo com elementos críticos.

Palavras-chave: intertextual, referências, Ludwig Hohl, Bergfahrt, persistente, teimoso, ascensão ao cume, caminhada, catalisador, tradição, transformar, romance, destacar, crítico

Unter dem Titel Dichter am Berg präsentiert der Bergsteiger und Schriftsteller Emil Zopfi seine Essays, in denen er kletternden und bergwandernden Schweizer Autoren nachsteigt und deren literarische Schilderungen mit eigenen Erlebnissen und Begegnungen verbindet. ${ }^{1}$ Er umgeht damit die klischeebehafteten Begriffe Bergroman oder Alpenliteratur, Stichwörter, die Hunderte von trivialen Heimat- und Liebesromanen frei geben, wenn man im Internet nach Bergliteratur Ausschau hält.

Dabei gibt es sie in reichlicher Auswahl, die Romane, Erzählungen und Filme, die es wagen, das traditionelle Genre auf unkonventionelle Weise anzugehen, die wohlbekannten Kulissen $\mathrm{zu}$ verschieben, die romantisierte Bergwelt $\mathrm{zu}$ verfremden und für aktuelle Problemstellungen nutzbar $\mathrm{zu}$ machen. Die Palette reicht vom aktuellen Interesse an historischen Ereignissen wie in Franz Hohlers Die Steinflut, Silvio Huonders Die Dunkelheit in den Bergen, einem ungelösten, vom Autor neu aufgerollten Kriminalfall, über kritische Stellungnahmen gegenüber tagesaktuellen Problemen der Region (Urs Mannharts Luchs) bis $\mathrm{zu}$ utopischen Zukunftsvisionen unter veränderten klimatischen und politischen Vorzeichen (Urs Augstburgers Wasserwässer, Christian Krachts Ich werde hier sein im Sonnenschein und im Schatten), um nur wenige, recht unterschiedliche Texte zu erwähnen. Der neue Alpenkriminalroman spielt in den labyrinthischen Gängen der sich im Bau befindlichen Alpentraversale, der Männerwelt der Mineure, in der die weibliche Ingenieurin neben den technischen Herausforderungen auch auf erhebliche mysteriöse Widerstände und ein Konglomerat von Vorurteilen stößt, wie dies beispielsweise die Staatswissenschaftlerin und Krimiautorin Sabine Altermatt in Bergwasser schildert ${ }^{2}$, oder 
es findet altes Unrecht, begangen an italienischen Gastarbeitern, bei neuen Projekten seinen Fortgang und wird geahndet (Urs Augstburger Schattwand).

Der Einbezug der Alpen in die Literatur verstärkt sich ab Mitte der 1970er Jahre und erweitert sich in den folgenden Jahrzehnten, als wäre es eine ausgemachte Sache zwischen Regisseuren, Filmemachern und Schriftstellern, die Bergwelt für die aktuellen Problemstellungen einzusetzen: Ältere und neue Romane werden verfilmt, neue Erzählungen geschrieben. Den Beginn macht Ludwig Hohls (1904-1980) Bergfahrt von 1975, die Erzählung, die den eigentlichen Auftakt zur Bergliteratur bildet, und worin die Frage gestellt wird: "Warum steigt ihr auf die Berge?" Sie wird beantwortet mit dem Satz:"Um dem Gefängnis zu entrinnen" (Hohl: 2014, 87-88). Bei Hohl repräsentieren die Berge die traditionelle Gegenwelt zum Tiefland mit den Bedingungen des Erwerbslebens und dessen Abhängigkeiten, stellen aber das Individuum vor die ultimative existentielle Herausforderung, worauf ich zurückkomme.

Weiter gelangen 1985 Meinrad Inglins Der schwarze Tanner in der Regie von Xavier Koller, die in Cannes ausgezeichnete Verfilmung Derborence von Francis Reusser nach Charles-Ferdinand Ramuz gleichnamigem Roman sowie Fredi Murers Höhenfeuer in die Kinos, Filme, welche die dunkle Ausstrahlungskraft von Berglandschaften für ihre Botschaft einzusetzen wissen. $^{3}$ Im Kontrast dazu, aber ebenso wirkungsstark, spielt das Filmkunstwerk des St. Gallers Peter Liechti Ausflug ins Gebirg (1986) auf den Alpenmythos an, lädt diesen aber negativ auf und bringt seinen ganzen Frust gegenüber der engen Bergwelt auf die Szene. Darin ist Liechti seinem St. Galler Journalist-Kollegen Niklaus Meienberg verwandt, dessen Schrift mit dem eindrucksvollen, einer naturwissenschaftlichen Dissertation abgekupferten Titel Die Erweiterung der Pupillen beim Eintritt ins Hochgebirge (1981) alles Schweizerische mit Mikroskop-Augen erfasst und einer sarkastischen Kritik unterwirft. Von Hermann Burger erscheint 1982 der Roman Die künstliche Mutter, in dem ihm das Glanzstück einer Umfunktionierung des HelvetiaMythos gelingt, der zugleich eine Rückführung auf den Alpenmythos und eine Verschmelzung mit ihm im Gotthard, dem schweizerischen Urgebirge, darstellt. Burger gestaltet"das Wasserschloss Europas" zu einem Venus- und umgestülpten Zauberberg, den 
er zugleich als groteske Militärbastion gestaltet, unterminiert von österreichischen Streitkräften, womit er den Mythos seiner Uneinnehmbarkeit schweiz- und armeekritisch zu brechen sucht.

Auch Franz Böni wäre hier zu erwähnen, sein Roman Die Alpen, die Erzählung Hospiz, ferner Ein Wanderer im Alpenregen, Texte, in denen die Alpen zu Alpträumen mutieren und zu Käfigen geworden sind. Damit hat das Gefängnismotiv, das wir bereits kennen aus Dürrenmatts Texten der 40er Jahre sowie aus Max Frischs Stiller, und das Dürrenmatt erneut einsetzt in seiner Havel-Rede von 1990, auch die Alpen erreicht, die bei den schärfsten Schweiz-Kritikern keine Alternative mehr bieten zur korrumpierten Welt der Gesellschaft im Flachland.

Wir sehen an diesen Beispielen die unterschiedlichen Funktionalisierungen der Alpen unter veränderten Vorzeichen: Während sie einerseits Freiheit und Unüberwindbarkeit und damit verbundene positive Werte versinnbildlichen, repräsentieren sie andererseits durch ihre Herausforderung Bedrohung und Lebensgefahr und können nicht zuletzt die sich selbst Überschätzenden in ihre Schranken verweisen. Diese Doppelheit hat die Auffassung der Alpen von Anfang an begleitet, weshalb ein kurzer Rückblick stichwortartig andeuten soll, wie der Gegensatz von Höhe und Tiefe, von Freiheit und Abhängigkeit zu den konstanten Wesensmerkmalen gehört.

Während die Wahrnehmung der Alpen von der Antike bis ins 15. Jhdt. vom Abscheu vor dem Fürchterlichen und Furchteinflößenden, von der Angst vor dem Unheimlichen und Gefahrvollen der Bergwelt verbunden ist, erfolgt im 17. Jahrhundert der Übergang von der theoretischen zur naturwissenschaftlich-praktischen Beschäftigung mit der Materie, die neben dem Fürchterlichen eine Anerkennung des Ästhetischen zulässt, das sich allmählich zum Erlebnis des Erhabenen entwickelt und im 18./19. Jahrhundert in der romantischen Periode den Höhepunkt erreicht. ${ }^{4}$

Literarischer Ausgangspunkt ist Albrecht von Hallers (1708-1777) mächtiges Gedicht Die Alpen von 1729. Es ist eine der ersten Lobpreisungen der Bergwelt, vor allem des Lebensstils der Bergbevölkerung, der zu dem der Bewohner des Tieflandes in starkem Kontrast steht. ${ }^{5}$ Wie wir wissen, wurde das Gedicht zur Initialzündung für das Interesse an 
den Alpen und den beginnenden Alpinismus. Grund dafür ist die darin zum Ausdruck gebrachte Bewunderung für die Vielfalt der sich in der Höhe manifestierenden Natur, welche die Furcht vor der Monumentalität und Unzugänglichkeit des Gebirges, dem rauen Klima und seinen Gefahren in den Hintergrund schiebt. Unten befinden sich"die verhassten Gründe”, auch das Vieh grüßt"den Berg mit Freuden"; unten ist"die Zivilisation mit tausend Büchern", oben wird"freudig durchgescherzt" (ein heute noch bestehender Unterschied: unten ist man per Sie, oben per Du). Unten ist der mühseligen Geschäfte und der Städte Rauch, oben stärkt das tätige Leben"der Leiber reife Kräfte / Der träge Müßiggang schwellt niemals ihren Bauch". ${ }^{6}$

Literarisch ist es Jean-Jacques Rousseaus Roman Julie ou La Nouvelle Heloïse (1761), der über 100 Auflagen erreichte und zur Weltliteratur gehört, der als erster die Verbindung von großen Empfindungen und leidenschaftlicher Liebe mit dem Erlebnis der Bergwelt verquickt. Auch er spielt den Kontrast Oben - Unten, Bergwelt - Flachland/Ebene aus, wenn wir hören, wie der Protagonist Saint-Preux in Julie die Wirkung beschreibt:"[...] der Berge senkrechte Ansicht rührt die Augen auf einmal und weit stärker als Aussicht auf Ebenen, die man nur seitwärts von fern sieht, und wo jeder Gegenstand uns einen anderen verbirgt. [...] Man ist da ernsthaft ohne Schwermut, ruhig ohne Unempfindlichkeit, zufrieden, dass man ist und denkt" (zit. nach Lesti, 2013: 40). Zwar spricht der Held, doch dahinter hören wir natürlich den Schweizer Rousseau, der die Vorzüge seiner Bergwelt anpreist. Doch wir lesen desgleichen bei seinem Zeitgenossen Carl Viktor von Bonstetten:"Kommen Sie: Hier sind Natur und Mensch frey und gross. Diese Wüsten, diese Felsen, diese Eisthäler, diese brausenden Alpenwasser, diese hohen schwarzen Wälder werden Ihnen besser gefallen, als die seelenlosen Schönheiten der Ebene" (zit. nach Schmidt 1990: 119).

Diese und weitere Veröffentlichungen lösen den Ansturm von bergsteigenden und erlebnissuchenden Briten, Franzosen, Deutschen und anderer Nationalitäten, sowie von Künstlern, Dichtern und Gottsuchern in die Schweizer Alpen aus, von Byron, Wordsworth, Mark Twain, Jens Baggesen, Friedrich Graf Stolberg, Goethe, Flaubert, Daudet, Conan Doyle bis hin zu Nietzsche, Thomas Mann und Hermann Hesse, welche ihre Erlebnisse in 
literarische Form bringen und ihre Leser zu Bergreisen inspirieren. ,Veni, vidi, Rigi' kann als Schlagwort über vielen dieser Reisen stehen, ${ }^{7}$ wobei Mark Twain mit seiner unglaublichen Schilderung einer Rigi-Bergtour zwecks Erlebnis des Sonnenaufgangs den Vogel abschießt und als Kronzeuge für die humorvoll-heiteren Gestaltungen dieses Genres stehen kann. Als modernes Beispiel einer Expedition mit tödlichem Ausgang sei Adornos gegen ärztlichen Rat ausgeführte Flucht zu seinem Traumberg Matterhorn (jedenfalls zu dessen Fuß) genannt, wo er einem Herzinfarkt erlag. Dies bringt ihn in die Nähe von Hans Castorp aus dem Zauberberg, macht Castorp zu einer Präfiguration von Adorno durch die Todesnähe, das Existentielle der Situation im Schneekapitel:"Allein finde ich nie und nimmermehr ins Flachland zurück" - so jedenfalls sieht es ein (ungenannt gebliebener) Schweizer Germanist, mit dem sich der Journalist und Bergsteiger Andreas Lesti unterhält (Lesti 2013: 220). Dass Thomas Manns Zauberberg ein äußerst zentraler Roman wäre unter dem Aspekt des Unterschieds zwischen dem Leben im Tiefland und dem in der Höhe (Hans Castorp ist für das Flachland verloren nach sieben Jahren Aufenthalt in Davos), soll hier erwähnt werden, ebenso Nietzsches Zarathustra und dessen Wanderungen - oder Nietzsche selbst, welcher der Höhenluft des Engadins die Inspiration für seinen Königsgedanken der ewigen Wiederkehr des Gleichen zuschreibt, doch sie liegen außerhalb des hier festgelegten Rahmens von zeitgenössischer Literatur aus der Schweiz.

Festzuhalten ist, dass sich neben dem Abenteuer- und Pioniertourismus, der in die Literatur Eingang findet, immer auch jene vertiefte Wahrnehmung der Erlebniswelt Alpen niederschlägt und in der Darstellung existentieller Selbst- und Grenzerfahrungen zum Ausdruck kommt, die oft ein Leben verändern.

Wie bereits angedeutet, werden die Alpen auch im 20. Jhdt. diversen ideologischen Richtungen dienstbar gemacht, einmal sind sie Ausdruck von reiner Naturverehrung oder von religiös verankertem Bergkult, von vaterländisch-gesinnter Heimatliebe oder radikaler Selbstverwirklichung oder sie wurden eingesetzt als Mittel radikaler Schweiz-Kritik und Entmythisierung. Nachdem der Zorn der Kunstschaffenden verebbt und sich die SchweizKritik gegen Ende der 1990er Jahre allmählich gelegt hatte, wurde der Zugang zu den Bergen literarisch wieder ein anderer. Das Augenmerk richtet sich nach 2000 vermehrt auf 
die Milieuprobleme, die negativen Seiten der Eingriffe in die Natur durch den rücksichtslosen Ausbau und die dominierenden ökonomischen Interessen, welche der Region Schaden zufügen im sozialen wie im kulturellen Bereich. Für einen enormen Anstieg der Bergliteratur sorgen auch die verschiedenen Bergjubiläen, die in den letzten Jahren gefeiert wurden, so die Erstbesteigung der Eigernordwand und die Erstbesteigung des Matterhorns. Sie veranlassen die Veröffentlichung einer großen Zahl von Dokumentarberichten, Filmen und Erzählungen, die neben allem Informationswert auch für Nervenkitzel und Spannung sorgen. Eine nicht zu unterschätzende Breitenwirkung haben auch die großen Tunnelbauprojekte wie die neue Alpentraversale, die Freigabe bestimmter Stollen des Réduits, die nicht nur zu besichtigen, sondern auch bewohnbar geworden sind, faszinierend und unheimlich in einem, gemessen an in ihren enormen Dimensionen und ihrer dunklen Vergangenheit. Die Kategorie des Furchterregenden mag sich zwar verringern, doch zeichnen sich neue Gefahren ab wie etwa die Klimakrise und mit ihr die fortschreitende Bedrohung des Lebensraums in den Bergen oder Bausünden und Abraumhalden, die Schäden verursachen. Kein Wunder, dass sich zahlreiche Schriftsteller und Regisseure mit diesen Umwelt- und Milieuproblemen sowie deren Auswirkungen auf die Menschen filmisch und literarisch auseinander setzen.

Ich gehe im Folgenden etwas näher auf zwei Romane ein, die sich von Ludwig Hohls Erzählung Bergfahrt inspirieren ließen und werfe dabei auch einen Blick auf Max Frischs Erzählung Der Mensch erscheint im Holozän von 1979.

Mit Urs Richles Mall (1993) erscheint ein Roman, der als Motto Ludwig Hohls schon zitierte Aussage trägt:"Warum steigt ihr auf die Berge?" “Um dem Gefängnis zu entrinnen.” Im Zentrum dieses Romans steht der Gonzen, der dominierende Berg von Sargans, in dessen Innern sich das größte stillgelegte Eisenbergwerk der Schweiz befindet, welches das Leben des Bergwerkingenieurs Mall ausgefüllt hatte. Er liegt auf der Pflegestation eines Spitals in Zürich, wo ihn der Ich-Erzähler Ulrich Hörmann pflegt bis zu seinem Tod und von dessen Arbeit im Berg in surreal anmutenden Gesprächen erfährt, weil sich in Malls Bewusstsein Gegenwart und Vergangenheit überschneiden. Der Pfleger wird völlig von Malls Geschichte absorbiert: 
Ich stelle mir nach und nach seine Geschichte her, seine Erinnerung, seine Person. Ich gehe mit ihm in den Berg, durch die Stollen, durch die Kanäle und Spalten, durch ein Labyrinth von Wegen und Gängen, von denen nur er zu wissen scheint, wozu sie führen, wozu sie nützlich sind.[...] "Über uns der Berg". (Richle 1993: 161)

Er verlässt seinen Posten am Tag nach Malls Tod und begibt sich nach Sargans, um dessen Spuren nachzugehen, mietet sich in einem Hotel ein, bleibt vier Monate weg, bis man ihn polizeilich abholt und in eine Nervenheilanstalt einliefert, völlig verwahrlost und verkommen. Er hatte zahlreiche Versuche unternommen, Wege auf den Berg auszukundschaften, ihn jedoch nie bestiegen trotz seiner befremdlichen Äußerungen, den Gipfel in direkter Route über die Steilwand $\mathrm{zu}$ bezwingen. Mit Hilfe einer Gesprächspartnerin werden die Gründe für Ulrichs Verhalten freizulegen versucht und er erzählt im letzten Teil des Romans von der Pflege des hochbetagten Mall, dessen Situation der eines in zweifacher Weise Gefangenen gleichkam: in seiner fragilen Körperhülle in seinem Spitalgitterbett liegend. Im Gegensatz zur Situation des Pflegers ist sein Geist aber frei, das Spital zu verlassen und in den Berg abzutauchen, während sich der Pfleger so sehr in das Schicksal seines Anvertrauten verstrickt, dass er auch nachts nicht mehr davon loskommt. Langsam wird die Geschichte des Ingenieurs bruchstückweise aus der Tiefe des Berges gehoben, der als schützende Hülle das zunehmend verschüttete Bewusstsein des sterbenden Patienten birgt. Eine erzählte Lebensgeschichte, in der neben einem Spital und einer Nervenheilanstalt ein abweisender Berg den Ort des Geschehens dominiert, der ausgehöhlt dem zu Ende gehenden Leben gleicht und doch sein innerstes Geheimnis über die Erzlieferungen im Zweiten Weltkrieg nicht preisgibt. So wie der Pfleger Malls Leben durch seine Geschichte erlebt hat, wollte er den Berg selbst erleben und hätte damit beinahe auch sein eigenes Leben zu Ende gebracht, weil ihm nur Unverständnis entgegenschlug und er sich daraufhin ganz auf sich selbst zurückzog. Vergraben in seinem Zimmer bezwingt er den Berg nur noch in seinem Bewusstsein, aber es ist ein sinnloser Sieg, weil er der Vergessenheit anheimfällt wie der Mensch. Doch auch dagegen wehrt sich der Pfleger: Für ihn ist Mall nicht tot, denn" [D]ieser Mensch in seiner ganzen 
Gebrechlichkeit war der Berg" (Richle 1993:103). Solange er sich die Freiheit des Denkens bewahrt und den Verstorbenen im Gedächtnis behält, bleibt er für ihn lebendig.

Der Gedanke an Max Frischs eindringlich geschilderten Ablauf des fortschreitenden Verfalls von Herrn Geisers Geistesfähigkeiten in Der Mensch erscheint im Holozän meldet sich, wo der Verfallsprozess der Gedächtnisstrukturen auf geniale Weise verknüpft ist mit der Entwicklungsgeschichte der Menschheit und den Anzeichen der zunehmenden Erosion der Natur durch den Klimawandel. Auch in Frischs Text von 1979 spielen ein Berg und eine Bergwanderung eine entscheidende Rolle, ebenso der Gegensatz Tiefland/Tal - Berggebiet (Herr Geiser wohnt eigentlich in Basel, verbringt aber seine Zeit in den Tessiner Bergen), denn die Gefahr von Bergrutschen ist ein Problem, das die Bewohner in ihren Siedlungen an den Hängen ihrer Bergtäler zunehmend beunruhigt. ${ }^{8}$ Die Wanderung über den Berg, zu der sich Herr Geiser bei schlechtem Wetter entschließt, ist eine Herausforderung, der er nicht mehr gewachsen ist, was er jedoch nicht abzuschätzen vermag. Ausgestattet mit den falschen Hilfsmitteln (Schirm statt Stock, Hut statt Thermosflasche) und von seinem Gedächtnis verlassen, versucht der 74-Jährige die Passhöhe, die ins Maggiatal hinüber führt zu erklimmen, um nach Basel zu kommen, ein Wunsch, seinem immer mehr einem Käfig gleichenden Haus und dem gefühlten Ende zu entfliehen. Die Expedition, normal eine zweieinhalbstündige Tour, kostet Herrn Geiser fast das Leben, weil Zeit- und Orientierungssinn sowie der Körper nicht mehr funktionieren. Die Überwindung des von Erosionen gezeichneten Hangs mit seinen Runsen und Schluchten bringt ihm seine Matterhorn-Tour ins Gedächtnis, die ihn und seinen Bruder in jungen Jahren fast das Leben gekostet hatte. Damals war es eine Fehleinschätzung, die sie auf die falsche Route und damit an den Rand des Abgrunds brachte. Das erfahrene Freiheitsgefühl verkehrte sich ins Gegenteil, die Situation wurde ausweglos, die Wanderer zu Gefangenen des Berges. Nur Zufall, dass das waghalsige Experiment des Bruders gelingt und die beiden aus der Wand fanden. 50 Jahre später sind die Dimensionen von einem Viertausender auf 1000 Meter geschrumpft, doch der Beweggrund für das Wagnis ist der gleiche, allerdings verursacht durch Demenz und Altersstarrsinn, die Folgen sind diesmal tödlich, denn Herr Geiser erleidet nach der Rückkehr einen Hirnschlag, es fehlt der rettende Bruder. 
Die von Frisch geschilderte Matterhorntour insinuiert durch die Genauigkeit der Wiedergabe der einzelnen Handgriffe und Kletterpartien die Tatsächlichkeit einer von Frisch selbst durchgeführten Besteigung des Matterhorns, die sich aber nicht belegen lässt. ${ }^{9}$ Sie erinnert aber auch in ihrer Präzision an die detaillierte Touren-Schilderung Ludwig Hohls in seiner Bergfahrt, die nicht nur Frisch inspiriert haben mag, sondern tatsächlich den jüngeren, in Berlin lebenden Schweizer Autor Roman Graf bei dessen Niederschrift des Romans Niedergang (2013, zit. N) beeinflusst hat. Das Problem der Gipfelbesteigung ist darin zentral und wird zum Katalysator für die Entlarvung von Denkweisen und Verhaltensmustern, die sich im alltäglichen Umgang hinter Konventionen verstecken. Es ist ein Buch, in dem es von Anfang bis Ende um einen bis in die Details der einzelnen Kletterhandgriffe hinein beschriebenen Aufstieg auf einen nicht näher bezeichneten Gipfel geht, der höher als 3500 Meter ist. Dass in diesem Kontrast (Aufstieg - Niedergang) eine intensive Spannung angelegt ist, die sich auf verschiedenen Ebenen entfaltet, bleibt dem Leser nicht lange verborgen, geht es doch um ein junges Paar, er Schweizer, sie Mecklenburgerin, beide in Berlin lebend, die diesen Ausflug mit einander unternehmen, den sie monatelang anhand von Karten detailliert geplant und mit Kletterkursen vorbereitet haben, weil André Louise seine Heimat zeigen und sie dabei auf eine ordentliche Bergtour mitnehmen will.

Dass das Wetter dann nicht mitspielt, ist zwar enttäuschend, soll aber echtem Enthusiasmus und gründlicher Planung keinen Abbruch tun. Zudem zeigt sich echte Könnerschaft am Berg gerade bei schlechtem Wetter, ist es doch keine Herausforderung, bei Sonnenschein und blauem Himmel einen Gipfel zu erklimmen: “... das schlechte Wetter muss man sich zum Verbündeten machen" (N:9), heißt es zu Beginn des Romans. Es ist ein Satz, mit dem sich André gegen besseres Wissen von der Richtigkeit seines Wunsches, die 5-tägige Tour trotz schlechten Wetters durchzuführen, selbst überzeugt. Er ist der Protagonist, aus dessen Blickwinkel erzählt wird, die Reflektorfigur, durch welche alles, auch das, was wir über Louise erfahren, vermittelt wird. Mit andern Worten haben wir es mit einem unzuverlässigen Erzähler zu tun, von dem wir nicht genau wissen, ob er Louise richtig auffasst oder missversteht. 
Was sich auf den ersten Seiten schon als leichte Verstimmung und Uneinigkeit andeutet, wird später zum Trennungsgrund und führt zur endgültigen Entfremdung zwischen den beiden. Louise hat keine Lust aufzubrechen und möchte schöneres Wetter abwarten, steuert auf eine Bäckerei zu und nervt den Freund mit dem langsamen Verzehr ihres Croissants. Später braucht sie Pausen, um sich an die Höhe zu gewöhnen, beladen mit einem bleischweren Rucksack, während es von André heißt: "Eine Wanderung, bei der es nicht steil aufwärts ging, war für ihn keine Wanderung” (N:17), wozu auch die Spaziergänge um die Seen Berlins und Mecklenburgs gehören. André, rücksichtslos sein Tempo haltend im hoffnungslos verregneten Grau von Stein und Nebeln, treibt sich selbst an, indem er sich an Härtetests aus seiner Pfadfinderzeit erinnert, aus deren Fehler er lernen musste, was ihn allerdings nicht befähigt, seine neuen zu erkennen und die nötigen Konsequenzen zu ziehen (die Mobiltelefone wurden zuhause gelassen, um die Natur umso genuiner zu erleben, für Schnee waren sie nicht ausgerüstet, der bei diesen Temperaturen in der Höhe nun fallen würde). Vor der Übernachtung in der Hütte mit dem Hüttenwirt aus dem Erzgebirge, mit dem sich Louise - sehr zu Andrés Ärger - ausgezeichnet versteht und ein reges Gespräch führt, während die beiden aneinander vorbei schweigen, sinkt die Stimmung bei André auf null, was ihn dazu bringt, seinen Ressentiments gegen Deutsches und Deutsche in der Schweiz freien Lauf zu lassen. Der Wetterumschlag mit Schneefall während der Nacht und Sonne am anderen Morgen bringt ihn aber zur Vernunft: er erkennt die Fehlplanung in Berlin und stellt auf eine leichtere Route um, welche beiden mehr Spaß an der Wanderung und größere Sicherheit garantieren soll.

Umgekehrt hat aber auch bei Louise ein Stimmungswechsel stattgefunden, bewirkt durch das Treffen mit dem freundlichen Deutschen und der strahlenden Sonne, so dass sie es während des mit Harmonie überschriebenen Kapitels ablehnt, die ursprünglichen Pläne für die Wanderung aufzugeben und auf die anspruchsvolle Route zu verzichten.”Der Gipfel lag versteckt, irgendwo hinten, weit in der Höhe. ,Wir müssen nach oben, sagte Louise, ich will hinauf!" (N:70). Endlich beginnt die richtige Wanderung, die alle Kräfte fordert und Louise überfordert, während André es genießt, an seine äußersten Grenzen zu kommen und sich selbst zu beweisen, dass der Wille das Entscheidende ist und über dem Verstand 
steht. Die ungewohnte Steinwüste, die Felsen setzen Louise physisch und psychisch zu, die mit Wanderungen um die Mecklenburgische Seenplatte vertraut ist, doch André fehlt jegliches Verständnis dafür, vielmehr fragt er sich,"weshalb er sich den Umgang mit diesem störrischen Esel aus Mecklenburg-Vorpommern antat" (N:107). "Auch er hatte sich an die flache Landschaft in und um Berlin, in Brandenburg gewöhnen müssen, was am Anfang nicht leicht gewesen war. Diese weiten Ebenen - eine desillusionierende Eintönigkeit" (N:112) und er kommt zum Schluss, dass ihm an dieser Wanderung einzig Louise nicht gefiel. Es ist zugleich der Punkt, wo Louise klipp und klar erklärt, dass sie nicht mehr weiter gehen mag und vor einer schwierigen Partie, die Teamwork erfordert, dem Durchklettern eines Kamins, den Rückweg antritt. Es ist die ultimative Kränkung, die sie André antut, die doch von ihm nicht verlangen kann, dass er das so lange und so minutiös geplante Unternehmen aufgibt (auch wenn er einige wesentliche Punkte übersehen hatte), u.a. die Hauptregel, nicht allein zu klettern. "Es musste sein.” “Die Erstürmung des Berges wurde wieder aufgenommen - oder die Flucht. André wusste nicht, ob er bloß einen Gipfel bestieg oder von Louise fortging" (N:124), heißt es. Vom Pfadfinder-Motto geleitet: Wo ein Wille ist, ist auch ein Weg" peitscht er sich durch Schneesturm und Felswände hoch, erreicht schließlich den Gipfel, seinen Olymp, erklommen trotz Schmerzen und unmenschlicher Anstrengung, doch der Triumph ist nicht wie erwartet: "wie ein Gott saß er da und schaute auf die Welt hinunter [...] er sah nur Dunkelheit" (N:177). Fatal war, dass er seine Aussteuer hatte liegen lassen müssen, was Übernachten und Abstieg fraglich macht. Er friert, ist verwundet und weiß nicht mehr, wie er wieder hinunter kommen soll: "Wie eine Katze fühlte er sich, die zu hoch auf einen Baum geklettert war und um Hilfe schrie" (N: 178). Er hätte sich jetzt sogar gern von jenem deutschen Hüttenwart retten lassen, dem er keine richtigen Kenntnisse der Schweizer Verhältnisse zugetraut hatte. Er vermisst Louise, fühlt sich verlassen und hat Angst vor dem Abstieg, für den er nach zwei Stürzen und Erfrierungen keine Kräfte mehr hat, leidet an Halluzinationen und entscheidet sich schließlich doch, ihn in Angriff zu nehmen: "Er wollte den Weg hinunter selber schaffen, und wenn es nicht gelang, wollte er sterben. So bliebe er wenigstens mit sich im Reinen" (N:178). Er kommt ins Rutschen, verliert jeden Halt und stürzt ab. Ob Louise den Abstieg 
geschafft und an den See gekommen ist zum gemütlichen Baden, wie geplant und wie Andrés Halluzination es ihm vorspiegelt, bleibt offen.

Als Apropos zu den Auseinandersetzungen zwischen den beiden über die Vorteile des Mecklenburgischen Flachlands kontra Andrés Bergwelt muss ich hier eine Kontroverse anführen, auf die ich stieß bei der Lektüre über die wissenschaftliche Beschäftigung mit den Alpen im 18. und 19. Jhdt., welche sich wie eine Basis zu den Querelen zwischen André und Louise ausnimmt. So stritten sich der Rostocker Naturwissenschaftler Georg Detharding und der Zürcher Gelehrte Johann Jakob Scheuchzer (nach beiden ist eine Straße benannt in Rostock und in Zürich) über die Luftqualität in den Alpen versus im Tiefland. In seiner Disputation von 1705 behauptete Detharding, dass die Luft im Gebirge so grob sei, dass die Alpenbewohner davon "ganz dumm" würden. Und er leitete das berühmte Heimweh der Schweizer von der Tatsache ab, dass sie die reine Luft der Niederungen nicht ertrügen, die sie ganz krank und elend mache. Das konnte der Zürcher Naturwissenschaftler Scheuchzer nicht auf der Schweiz sitzen lassen und er schrieb: Niemals könne das Heimweh von "unserer langen Gewohnheit an eine unreine/innert den Bergen eingeschlossene Luft" herrühren. Das könne nur der Fall sein "bei einem Rostocker/oder Holländer/ wann der aus seiner dichten unreinen Seeluft" in die Schweiz komme und "da unsere ohne Zweifel reineste Luft von Ganz Europa in sich schlucken" würde. Im Tiefland finde "eine allzustarke zusamen Trukung sonderlich der kleinsten Blutgefässen [...] statt, welche den Kreislauf der Säfte hindere, was aber nicht in der subtilen Luftspher" der Alpen geschehen könne. Das Heimweh der Söldner sei unbestritten, habe aber andere Gründe, so die ungewohnte, strenge Kriegsdisziplin, meint Scheuchzer in seiner Antwort auf Detharding. ${ }^{10}$

Unabhängig davon, ob Graf von dieser Kontroverse Kenntnis hatte oder nicht, zeigt sich die Kontinuität des Diskurses Berge - Tiefland. Graf macht aber in einem Gespräch klar, dass sich der Gegensatz Flachland-Metropole - Alpenwelt so ergeben habe, jedoch nicht das Zentrale für ihn sei. Ihm sei es darum gegangen zu zeigen, wie eine Landschaft Menschen und ihr Denken präge. ${ }^{11}$ Er selbst lebt seit 10 Jahren in Deutschland, 3 davon in Leipzig (Literaturinstitut), wo er lernen musste, die Flachheit auszuhalten. Die totale Rundsicht bis an den Horizont ist für ihn Realismus pur, während seines Erachtens der Schweiz mit ihren 
Hügeln und Bergen etwas fast Märchenhaftes anhaftet, weil jederzeit Überraschungen möglich seien. Das Denken hingegen empfindet er dort als kleinräumiger gegenüber der Großräumigkeit in Deutschland, die er schätzt und die auch in der Mentalität zum Ausdruck komme.

Das Denken Andrés ist von dieser Kleinräumigkeit geprägt und nimmt im Verlauf der Handlung immer extremere Züge an. Seine an sich positiven Charaktereigenschaften wie Zielbewusstsein, Zielstrebigkeit entwickeln sich auf dem Aufstieg zu sturer Rechthaberei und Uneinsichtigkeit, gegen die Louise nicht mehr ankommt. Je fester er alles im Griff haben will, desto mehr entgleitet ihm die Voraussetzung des Gelingens für Tour und Partnerschaft. So kommt es zur Auflösung der Beziehung schon vor den schwierigsten Partien. Wut und Trauer über den Verlust der notwendigen Bergpartnerin streiten sich anschließend in André: Er muss den Gipfel erreichen ohne Louise, die ihn nun nicht bewundern kann in seiner wahnhaften Ambition, die ihn (so findet er) als künstlerischen Menschen kennzeichnet, der ein hohes Ziel anstrebt und dies mit Einsamkeit bezahlen muss. Bei dieser Selbsteinschätzung von André sind wir wieder bei Nietzsches Gedankengut.

Stark ausgeprägt findet sich dieses Motiv in Ludwig Hohls Erzählung Bergfahrt (zit. B), die 1975 herauskam und als ein Klassiker der Schweizer Bergliteratur gilt. Sie erzählt von der Bergtour zweier ungleicher Freunde, die sich entzweien und die mit deren Tod endet. Graf fiel das Buch während einer Schaffenspause in die Hand, ein Glücksfall, wie er meint, denn er entdeckte viel von sich selbst darin. Es ist die Parabelform, die Graf inspiriert und die er auf die heutige Zeit anwenden wollte. Während bei Hohl ein dominanter auktorialer Erzähler die Darstellung dominiert und für die beiden Bergkameraden, den zielstrebigen, rücksichtslosen Ull und den unschlüssigen, zögerlichen Johann spricht (wie denn dem ganzen Sprachduktus etwas Archaisch-Widerständiges anhaftet, das sich vielleicht auch damit erklären lässt, dass Hohl die Erzählung schon 1926 geschrieben, sie dann aber immer wieder bearbeitet hat, wie er das mit allen seinen Texten tat), macht Graf André zu einem personalen Erzähler. Sein Bewusstsein ist der Filter, durch den wir alles vermittelt bekommen, auch was Louise betrifft, so dass wir nicht wissen, ob 
wir ein wahres, ein leicht manipuliertes oder gar ein unrichtiges Bild von ihr erhalten. Die hauptsächliche Vermittlung seiner Gedanken erfolgt durch erlebte Rede, die gegen Ende auch in ein Gespräch mit der abwesenden und immer stärker vermissten Partnerin Louise übergeht. Die Unterschiede zwischen Hohl und Graf, die neben unglaublich faszinierenden Interferenzen wie Motivparallelen und Handlungselementen unübersehbar sind, liegen im Unterschied der Figuren (zwei ungleiche Bergkameraden bei Hohl, der eine lang und dünn und schlaksig, der andere kurz, zielbewusst, ja stur und durchtrainiert, ein Willensmensch mit einem hohen Ziel vor Augen, und darin ein Künstler. (Eine Freundin taucht nur auf als Imagination in der äußersten Gefahr, als sich ein Wunsch um Hilfe bei Ull einschleicht.) Keiner von beiden überlebt: Johann nicht, obwohl er, der Willensschwache, die Tour abbricht, den Freund allein lässt und umkehrt, denn er ertrinkt unspektakulär, indem er der Tücke eines Wildbachs zum Opfer fällt, dessen Gefahr er unterschätzt. Ebenso wenig Ull, der im Alleingang seine Grenzen überschreitet und das Erklimmen des Gipfels, den Sieg über den Berg, mit dem Tod beim Abstieg bezahlt, im Kampf mit dem Berg also doch unterliegt. Partnerschaft am Berg erweist sich als überlebenswichtig, nicht allein zu gehen, gehört zu den Grundregeln. Sie rettet den jungen Geiser in Holozän, der mit seinem Bruder unterwegs ist; der alte Herr Geiser hingegen ist allein und entsprechend der Natur und dem Berg ausgeliefert. Alleingang und Selbstüberschätzung sind schlechte Eigenschaften für Kletterer, die sich und ihrer Umwelt sich selbst beweisen wollen. Sie sind verantwortlich für das Versagen und den Tod in den genannten Erzählungen. ${ }^{12}$

Graf hat aus den Bergkameraden bei Hohl eine Paarbeziehung gemacht und trägt der globalisierten Welt Rechnung, indem er die beiden aus unterschiedlichen Ländern zusammenführt und sie mit unterschiedlichen Mentalitäten ausstattet, welche durch die Probe dieser Bergtour unter schlechten Bedingungen auf die Probe gestellt werden. Bei Hohl findet Ull kurz vor seinem Tod die endgültige Antwort auf die Frage: "Warum steigt ihr auf die Berge? ... Dies war es: Um dem Gefängnis zu entrinnen”. Doch dann heißt es “...Und nun?" (B: 87-88). Was ist an seine Stelle getreten? Ein neues Gefängnis? Auch der Berg kann seinen Bezwinger zum Gefangenen machen indem er ihn nicht mehr lebendig freigibt aus der Wand, der Schrunde, dem Kamin. Letztlich ist er der Gefangene seiner selbst, seiner 
heroischen Selbstüberschätzung, die er nur durch teuer erkaufte Einsicht in die Lebensgefahr überwinden kann, wenn er denn überlebt wie im Falle von Frischs Balz Leuthold in der frühen Erzählung, oder aber er bezahlt mit dem Tod, den er zum Befreier erhebt.

Hohls Erzählung ist stark autobiographisch geprägt: Wie sein Held Ull arbeitete er selbst minutiös und monatelang die Pläne zu seinen Bergtouren aus, führte sie bei schlechtesten Wetterbedingungen aus, um sich zu beweisen und riskierte dabei öfters sein Leben. Auch die Uneinigkeit mit dem Bergkameraden, der aufgibt und ihn allein weiterklettern lässt, ist selbst erlebt. Das Erringen des Berggipfels hat für Hohl metaphorisch die Bedeutung, als höchstes Ziel das Künstlertum und damit die absolute Freiheit zu gewinnen, und es war eine solche Bergtour, auf der er sich für den Dichterberuf entschied, der dann allerdings eine recht merkwürdige Form der Freiheit annahm, da er viele Jahre seines Lebens in recht eigenbrötlerischer Weise in einer Kellerwohnung in Genf verbrachte.

Auch Graf hat autobiographische Elemente in seinem Roman verarbeitet, das Motiv des Auswanderns und der Rückkehr, das Kennenlernen anderer Sehweisen und Koordinaten. Er braucht die Distanz zur Schweiz, zur Bergwelt, und die Freiheit der Großstadt Berlin, um schreiben zu können - und ist damit nicht allein, denn nicht wenige andere Schweizer Schriftsteller leben und schreiben in der Gegend von Berlin und Brandenburg.

Um zu zeigen, wie Roman Graf respondiert auf Ludwig Hohls Schilderungen, zitiere ich die Schilderung des imposanten Anfangsbildes aus der Bergfahrt und stelle den Abschluss von Roman Grafs Niedergang daneben.

Das erste Kapitel der Bergfahrt trägt die Überschrift Ein ungeheures Schiff und meint damit das Gebirgsmassiv, das sich auf dem unermesslich weitem Sockel aus dem Meer des Grünen zweier Täler erhebt. Zuoberst liegt der langgezogene höchste Grat,

[...] der jählings in fast senkrechtem Abbruch endet; der, mehrere Gipfel verbindend, kampflos, makellos vor dem hellen, flimmernd hellen Hintergrunde sich hinschwingt, den größten Gegensatz darstellend zu jedem zerklüfteten, wilden, bizarren Gebirge. 
So ist der Berg aus einer gewissen Distanz und von unten gesehen. An ihm ist nur Steilheit, gelassener Sieg, fragloser Sieg. Der oberste Teil seiner Flanke [...] gleicht einem Schild, einem Panzer, einer feinen, in Stahl und Silber eingelegten Arbeit. Und das ganze langegezogenen Gebilde dieses Gipfelbaues vor den hellen Himmeln hätte vielleicht auch den Eindruck erwecken können von einem sehr großen Schiff, das nicht in ein Erdenmeer nur, das in die Ewigkeit hinein führe. (B:9)

Schon im Anfangsbild ist der Sieg als fraglos vorausgesetzt, die Attribute von Steilheit, Schild und Panzer weisen auf die Stärke seiner Kampfmittel, die den Kampf von vornherein als überflüssig erscheinen lassen (kampflos, makellos).

Der Niedergang beginnt mit einem der sentenzhaft eingehämmerten Gedanken Andrés: "Am höchsten hinauf, am weitesten kommt, wer mit der Natur verschmilzt; das schlechte Wetter muss man sich zum Verbündeten machen" (N:9), und endet wie folgt:

Und André fiel. Flog rückwärts durch die Luft, Meter für Meter, Sekunde um Sekunde, so viel Zeit verstrich, und schlug endlich auf. Der Oberschenkel schmetterte auf Eis und wurde zu Eis. Der Rücken schlug auf Schnee und wurde zu Schnee. Wie ein Papierschiffchen, das einen Wildbach hinuntersauste, drehte er sich, kenterte; er wurde unter Schnee gedrückt. Alles begann langsamer zu werden. (N: 205)

Dem ungeheuren Schiff des Bergs gegenüber verhält sich der Mensch wie ein kleines Spielzeug-Papierschiffchen, das ihm ausgeliefert ist. Den Preis der Einheit mit dem Naturriesen (Eis und Schnee) bezahlt er mit dem Leben. Ob es sich gelohnt hat? Die Antwort von Hohls ganz durch seinen Ehrgeiz bestimmten Protagonisten wäre wohl ein vorbehaltloses Ja. Grafs André hingegen durchlebt in der letzten Phase seiner aussichtslosen Manöver über dem Abgrund die Erfahrung von Verlassenheit und Angst und "wollte diesen Sieg nicht mehr. Er gäbe ihn zurück und mit ihm sämtliche Medaillen und Trophäen, wenn er nur wieder unten bei den Menschen, einer von vielen sein könnte" (N: 202). Und trotzdem ist sein letzter Gedanke im Todesaugenblick reflexartig der, "dass er aufstehen und weitergehen müsse, wie beim letzten Sturz. Dann sah er Gämsen, zwei Gämsen, die an einem See standen, irgendwo auf Louises Seenplatte. Majestätisch senkten sie die Köpfe zum Wasser, und sie tranken" (205). Und mit diesem Bild vor dem inneren 
Auge löst sich der Gegensatz zwischen Flachland und Bergen in der Idylle auf, aber zu spät für die beiden.

\section{Bibliografie}

Altermatt, Sabina (2014), Bergwasser, München, Piper.

Augstburger, Urs (2014), Schattwand. Ein Bergdrama, München, dtv [bilger 2001]

-- (2012), Graatzug. Ein Bergroman, München, dtv [bilger 2007]

-- (2012), Wässerwasser, München, dtv [bilger 2009].

Barkhoff, Jürgen/ Valerie Heffernan (Hg.) (2010), Schweiz schreiben. Zur Konstruktion und Dekonstruktion des Mythos Schweiz in der Gegenwartsliteratur, Berlin /New York, de Gruyter.

Baumberger, Christa (2014),"Mit fremden Augen durch Berlin." Gespräch mit Stefanie Sourlier und Roman Graf, in viceversa literatur 8, Zürich, Rotpunktverlag S. 49-56 (= Jahrbuch der Schweizer Literaturen, Fokus"Berlin - mein Ding")

Böni, Franz (1979), Ein Wanderer im Alpenregen, Erzählungen, Frankfurt/M., Suhrkamp.

-- (1980), Hospiz, Erzählung, Frankfurt/M., Suhrkamp.

-- (1983) Die Alpen, Frankfurt/M., Suhrkamp.

Del Buono, Zora (2015), Gotthard. Novelle. München, C. H. Beck.

Frisch, Max (2009), Antwort aus der Stille, Frankfurt/M., Suhrkamp [1937].

$\begin{array}{lr}\text { N. }{ }^{3} 34-06 / 2016 \text { | 409-429 - ISSN 1645-1112 | http:/dx.doi.org/10.21747/16451112/litcomp34a25 } & 426 \\ \text { INSTITUTO DE LITERATURA COMPARADA MARGARIDA LOSA | WWW.ILCML.COM } & \text { CADERNOS DE } \\ \text { LITERATURA COMPARADA }\end{array}$


-- (1979), Der Mensch erscheint im Holozän, Frankfurt/M., Suhrkamp.

Graf, Roman (2015), Niedergang, München, btb [2013].

Haller, Albrecht von (1729), Die Alpen, in http://gutenberg.spiegel.de/buch/die-alpen$5457 / 1$

Hohl, Ludwig (2014), Bergfahrt, Frankfurt/M., Suhrkamp [1975].

Hohler, Franz (2008), Der neue Berg. Roman, München, Luchterhand [1989].

-- (1998), Die Steinflut. Eine Novelle, München, Luchterhand.

Huonder, Silvio (2014), Die Dunkelheit in den Bergen. Roman, München, Nagel \& Kimche.

Kracht, Christian (2008), Ich werde hier sein im Sonnenschein und im Schatten. Roman, Köln, Kiepenheuer \& Witsch.

Mannhart, Urs (2004), Luchs. Roman, Zürich, Bilger.

Lesti, Andreas (2013), Oben ist besser als unten. Eine literarische Expedition in die Alpen, Berlin, Rogner \& Bernhard.

Meienberg, Niklaus (1981), Die Erweiterung der Pupillen beim Eintritt ins Hochgebirge, Zürich, Limmat.

Obschlager, Walter,"Man, Culture, and Nature in Max Frisch's Der Mensch erscheint im Holozän", in Olaf Berwald (Hg.) (2013), A Companion to the Works of Max Frisch, Rochester/New York, Camden House, S. 197-210.

Richle, Urs (1993), Mall oder Das Verschwinden der Berge, Berlin, Gatza.

Schenk, Thomas (2010), Im Schneeregen. Eine Geschichte, Frankfurt/M., Suhrkamp.

Schmidt, Aurel (1990), Die Alpen - schleichende Zerstörung eines Mythos, Zürich, Benziger.

Werner, Markus (2004), Am Hang. Roman, Frankfurt/M., Fischer.

Zopfi, Emil (2009), Dichter am Berg. Alpine Literatur aus der Schweiz, Zürich, AS Verlag.

Beatrice Sandberg, Studium der Germanistik, Anglistik und Nordistik an den Universitäten 
Zürich und Bergen, Promotion in Zürich. Anschließend Dozentin und 1984-2012 Professorin für deutschsprachige Literatur an der Universität Bergen. Veröffentlichungen zur deutschsprachigen Literatur des 19. und 20. Jh.s (C. F. Meyer, Franz Kafka), zu rezeptionsgeschichtlichen Themen, zu Literatur und Faschismus, zu Fragen der nationalen und kulturellen Identität und zur Schweizer Literatur (Friedrich Dürrenmatt, Max Frisch, Erica Pedretti, Peter Bichsel, Urs Widmer, Gerold Späth, Gertrud Leutenegger, Thomas Hürlimann, Silvio Blatter, Binjamin Wilkomirski, Irena Brežna u.a.).

\section{Fussnoten}

1 (2009) Alpine Literatur aus der Schweiz, Zürich, AS Verlag. Ich begrenze mich in meiner Darstellung ebenfalls auf Beispiele aus der Schweiz.

2 Auch Zora del Buono, Bauleiterin und Architektin, macht in der Novelle Gotthard (2015) den Gotthardbasistunnel zum Gegenstand ihrer Kriminalgeschichte.

3 Urs Augsburger integriert Literatur über Walliser Brauchtum und Dokumentarliteratur zum Bau der Staumauer Grand Dixence in seinen Kriminalroman Graatzug von 2007, der auf jahrelangen Recherchen basiert und handfeste Realität mit Alpenmystik auf spannende Weise verbindet.

${ }^{4}$ Ich stütze mich auf die reichhaltige Studie von Aurel Schmidt (1990), Die Alpen - schleichende Zerstörung eines Mythos, Zürich, Benziger, welcher die Vielfältigkeit der Alpen in ihrer Entwicklung nachzeichnet und viele literarische Bezüge anführt, aber auch auf die bedenklichen Konsequenzen einer schleichenden Zerstörung des Alpenmythos aufmerksam macht. Einen „Weg der Bücher durch die Alpen“ vermittelt Andreas Lesti in: Oben ist besser als unten. Eine literarische Expedition in die Alpen, Berlin, Rogner \& Bernhard 2013). Hier finden sich weitere auch nicht-deutschsprachige Beispiele. 
5 Das geschieht noch in antikischer Manier. Genaues darüber bei Hans-Jürg Schrader: „Hallers Die Alpen, mythenkritisch reflektiert in Brandstetters Almträume“, in Barkhoff/Heffernan 2010: 77-93.

${ }^{6}$ Zit. nach: http://gutenberg.spiegel.de/buch/die-alpen-5457/1

${ }^{7}$ So die Überschrift eines Kapitels bei Lesti (2013:160). Vgl. auch die dort wiedergegebene, unsäglich witzige Schilderung von Mark Twain (160-165).

${ }^{8}$ Relevant in diesem Zusammenhang ist die frühe, später von Frisch verworfene „Erzählung aus den Bergen“ Antwort aus der Stille von 1937, die stimmungsmäßig überfrachtet ist, aber von einem jungen Mann handelt, der sein Leben riskiert, als er im Alleingang einen unbezwungenen Berggipfel besteigen will und mit Erfrierungen zurückkommt. Er musste erfahren, wie viel stärker als der Mensch die Natur ist. W. Obschlager weist darauf hin, dass auch in Frischs journalistischen Arbeiten der 1930er Jahre die Berge einen zentralen Platz einnehmen (2013:198).

${ }^{9}$ Vgl. dazu die Ausführungen bei Zopfi (2009: 244-247).

${ }^{10}$ Der Gelehrtenstreit und die daraus zitierten Stellen sind referiert bei Schmidt (1990), S. 62-63.

${ }^{11}$ Siehe dazu das Interview mit Stefanie Sourlier in: viceversa literatur 8 (2014: 49-56).

12 Ähnlich gelagert ist ein „harmloser Ausflug“ des Bankers Schwitter in der brillanten Erzählung Schneeregen (2010) von Thomas Schenk, der trotz Temperatursturz und Schneefall im Alleingang weitergeht und sich im Spital wiederfindet, aus dem er kaum mehr herauskommt.

N. 34 - 06/ 2016 | 409-429 - ISSN 1645-1112 | http:/dx.doi.org/10.21747/16451112/litcomp34a25 